\title{
Programming experiments on a TERAK 8510/A microcomputer
}

\author{
TIMOTHY A. POST and JAMES L. FOX \\ Learning Research and Development Center, University of Pittsburgh, Pittsburgh, Pennsylvania 15260
}

\begin{abstract}
In the course of bringing up one's first experimental microcomputer system, certain standard problems occur that are trivial to the experienced programmer but are great blocks to the novice. In this spirit, some paradigms that were implemented on a TERAK 8510/a microcomputer, and the related problems, are described. Specific topics include some consideration of timing procedures for reaction time experiments and how to handle large text files on small floppy disk systems.
\end{abstract}

In the course of writing code for a particular program, certain obstacles are met that require unusual or "tricky" solutions. A complicated calculation may be necessary to generate random numbers, or a sophisticated algorithm may be needed in order to traverse a binary tree. Further, as we write more and more programs that are similar in intent, we often find ourselves using and reusing some of the "tricks," even duplicating the exact code. At a more general level, patterns of code can be used to facilitate learning of a language (Soloway \& Woolf, Note 1) over the more traditional one-line definition approach of many computer books (see Grogono, 1980 , for an example in contrast).

This paper presents some typical problems encountered in the course of programming experimental applications. The methods, which work in our current applications, may be helpful to others.

\section{Task Descriptions}

The programming presented here was designed for collecting reaction time data for a knowledge-base text processing study. All aspects of stimulus presentation, response measurement, and data recording were to be performed by a microcomputer for ease and increased accuracy. More specifically, three major data gathering programs were developed: CONMEM (an acronym for continuous memory), which presented large texts, one sentence at a time, with the ability to test recognition of stimulus sentences; WORDID (word identification), in which words and short phrases were presented on the screen in a word identification task; and SBERG (Sternberg), which was an adaptation of the letter identification task employed by Sternberg (1969) (the adaptation involved using sentences as the basic units

This report was supported by the Learning Research and Development Center, supported in part as a research and development center by funds from the National Institute of Education (NIE), United States Department of Education. The opinions expressed do not necessarily reflect the position or policy of NIE, and no official endorsement should be inferred. of analysis, rather than letters). A fourth program, TCH, was used for introducing subjects to the apparatus and collecting some basic reaction time data. TCH involved a two-choice reaction time decision.

CONMEM was written to collect data on working memory processing and structure in domain-specific texts by subjects who either knew little or a great deal about the domain. The basic task involved the presentation of a main text, one sentence at a time, for the subjects to read. Periodically a message on the screen informed the subject that a test trial was about to occur. This allowed the subject to prepare to respond to the test item by pressing one of two keys on the computer keyboard. Four variants of the task were programmed. The first version had the subjects receiving different stimulus sets, depending on the condition under which they were to be tested. The stimuli were tested at the same points in the main text. This prevented the betweensubjects manipulation of the interval between probe item (within the main text) and test item, so the second variant was constructed with this capability. The third version changed the nature of the main text presentation from a fixed rate (usually $5 \mathrm{sec} /$ sentence) to a subjectpaced format, with the subject demand time written out to the data file. The fourth version of this program involved taking the second version and adding context capability; that is, the appearance or lack of singlecontext items preceding each test sentence could be varied for specific probes between subject sessions.

The WORDID program presented blocks of 40 trials. Each trial had two forms: a simple word identification (this took the form of a yes/no response to whether a word belonged to a certain domain or category) and the same method with a fixed-duration context word or phrase preceding the test item. The form of presentation, whether with or without context, was determined by the structure of the stimulus file and did not change during a particular session. The SBERG program presented a short text of a variable number of sentences, analogous to the memory set manipulation used by Sternberg (1969). This was immediately followed by a stimulus, 
and the subject had to determine whether or not its content presented information that had appeared during a previously presented scenario. Finally, the TCH program presented a " $\mathrm{Y}$ " or an " $\mathrm{N}$ " in 1 of 10 screen locations that were roughly equally spaced about a central point. Each letter presentation was preceded by a fixation point of variable duration, and subjects pressed a key marked " $Y$ " if the stimulus was " $Y$ " and " $N$ " if it was "N." Either 60 or 30 trials were presented to the subjects, depending on the experiment in which they were participating.

\section{System Description: Hardware}

The microcomputer used for the above experiments is a TERAK Corporation Model 8510A. This system has three components: processor and disk, keyboard, and display unit. The processor incorporates a DEC LSI-11 unit, $28 \mathrm{~KB}$ words of 16-bit RAM and hardware arithmetic processing. A soft-sectored floppy disk unit is provided within this unit as part of the basic system. The video control circuitry is also contained within this piece. This circuitry had two display buffers, one for 24 lines of 80 characters and the other for 240 by 320 graphics dot resolution. The display unit stands on a fixed pedestal and has a 12 -in. black-and-white viewing screen. A 71-key grouping with cursor-control keys and a numeric pad make up the keyboard. This system has been very complete and reliable. The only alteration to this system was the addition of a hardware clock capable of millisecond timing resolution. The clock used one of the two available device ports on the backplane provided on the Terak. A fuller description of the clock attributes and the programming involved in accessing it is contained later in this paper.

\section{System Description: Operating System}

The software package used with this system is UCSD PASCAL. Detailed evaluations of PASCAL appear elsewhere (e.g., Fox, McKnight, \& Wolf, 1981). A brief description is provided here.

UCSD PASCAL provides three major advantages. As a system, it is very complete. In addition to its program compile, link, and execution capabilities, the support software (e.g., file maintenance and editor) is very sufficient for most applications. Further, because of its use of a pseudomachine concept, the entire system can and has been implemented in many different systems. A project currently being undertaken involves the adaptation of the transferable version of UCSD PASCAL for implementation on microcomputers that previously have not had UCSD PASCAL adaptations. An attempt is being made to develop a standard basic input/output system (SBIOS) for the a dap table UCSD PASCAL system that can be written in a relatively small amount of read. only memory. This capability will allow for the design of small and inexpensive microcomputers that use a larger mainframe computer as a pseudodisk (see Fox et al., 1981, for these details). In making this system diverse enough on 6800 microprocessors, one can add priority capability. This concept of widespread system usage leads to the second advantage: transferability of software. Through portability at the system level, we are provided a framework for the development of software for use on the particular package. This standardization, coupled with similarities in objectives, can aid in the trading of software among individual users. The interchange of software between systems can reduce the overhead involved in the development of software for laboratories; the advent of networks among such groups can also increase such interaction. Finally, PASCAL as a programming language can lead to programs that are structured in nature. This allows for better breakdown of problems into tractable designs and greater ease in understanding of code, according to structured programming proponents. Since the language was developed for learning general programming concepts, it is also easy to learn.

UCSD PASCAL is not without disadvantages, however. First, a knowledge of how the system performs certain functions can always lead to a fuller understanding of it: since this information is sometimes lacking, certain tasks can be hindered, such as installation on new systems. The most likely reason for this problem is the proprietary nature of this software package (i.e., the owners wish to protect their product). Another slight difficulty with UCSD PASCAL is the file structure used for its disks. This variant can make it difficult for reading a disk by another operating system. Despite these minor inconveniences, sufficient methods exist for achieving the programming goals that are typically needed.

The remainder of this paper cites some stumbling blocks encountered during the course of programming the above-mentioned programs.

\section{TIMING CONSIDERATIONS}

A problem that has plagued programmers of experimental systems is how to acquire accurate timing with sufficient resolution. Both of these concerns are especially critical in reaction time situations. Any source of noise in data, such as inaccurate timing, is intolerable, for the amount of variance both within the between subjects is already quite high. Likewise, the resolution of clock matters when the effects that are sought are small in magnitude. (A more technical treatment of timing problems can be found in Christian and Polson, 1975.)

There are three general solutions to this problem that can be outlined, but two are not satisfactory for our purposes. The first method involves the use of a software clock at the assembler level of programming. This requires the knowledge of the cycle times for individual instructions that will be executed for a fixed loop of code. Given this information, the amount of time per loop can be counted and multiplied by the number of loops executed. While this method is suspect with respect to accuracy (the debate here is that a microcomputer's cycle time may be more variable than 
desired), the main reason for rejecting this approach is the lack of awareness of what the system does at the system level, especially with disk maintenance. While software clocks have been applied successfully in the past (e.g., Post, 1979), the users usually have had access to knowledge about control when the system had interrupts pending. In the TERAK system, the way that the system performs many activities is not specified in the documentation provided, so this route was not chosen.

A real-time clock might be desirable. One is provided by the manufacturer in the form of a line clock that synchronizes with the start of vertical retrace. This solution circumvents the problems involved with software clocks, but it is not adequate for our purposes. Since this clock is dependent upon the input power, its resolution is limited to $16.7 \mathrm{msec}$. While we were not sure of exactly how small and variable our desired effects were going to be, previous studies led us to believe that they would be minute and fragile. Errors of plus or minus one clock tick could produce an unreliable difference of $33 \mathrm{msec}$, and further resolution was desired.

To this end, we decided to have a hardware clock that was capable of millisecond accuracy. One such device, MCLOCK, was designed and build within our laboratory for use with a microcomputer.

\section{MCLOCK Description}

Under program control, the laboratory-built clock unit can be loaded with a value from 0 to 65535 , counted up or down. Further, the unit can be read as it counts and can generate an interrupt when it passes through zero (this provides the capability of timing for durations greater than $65 \mathrm{sec}$ ). This circuitry is very reliable, now that it has been debugged.

The clock software is written at the assembler level. This function, MCLOCK, has four basic responsibilities: (1) The clock can be turned on (i.e., a countdown value can be loaded in and the clock will be started). (2) The clock can be read "on the fly," which actually means that the clock will be paused, read, and restarted, which throws the future timings off by a small amount of software overhead. (3) It can be turned off. (4) An interrupt routine handles the passing-through-zero event. This software maintains a real count, in milliseconds, of how long the clock has been running.

There were two major difficulties with the programming of MCLOCK: maintaining the real value of the elapsed time and returning this value to PASCAL via the stack. The first problem was just a matter of realizing that we needed to subtract the current value in the hardware clock from the loaded value and add the result to the stored real value (which amounted to the number of interrupts received times the loaded value) to get the current elapsed time. This problem was complicated by our trying to use assembler language. The difficulty involved with the stack was solved by our fully understanding the stack protocol. When performing a function call, the TERAK system pushes either the scalar argument values or the variable addresses on the stack from left to right, so that once the assembler routine is entered, the rightmost argument is the first item to be popped off the stack. When returning, all 32 bits of the floating-point timing value were pushed onto the stack after all of the arguments had been cleared (the return from the routine was accomplished via a register rather than stack; either method is viable).

While MCLOCK is capable of much more than is needed for our application, the use of the clock at the PASCAL level is quite straightforward. Since our latencies are not generally large, the option for interrupts is overridden by loading a large countdown value. The general method is as follows: First, the screen is set up with a trial. Then, the clock is started by a call through the MCLOCK assembler routine. Next to appear in the PASCAL code is a "read(keyboard,ch);", which simply holds the system until a key is struck on the keyboard. Two other steps are then taken. First, the clock is read in order to obtain the latency, which has a slight and fairly constant error added to it due to system overhead. Finally, the MCLOCK PASCAL assembler function is called with the parameter instructing the clock to be turned off. This is done to circumvent a curious problem. Since the interrupt service routine is not stored in a section of system RAM, after the program that has used the MCLOCK has terminated, the interrupt routine is erased. After the time that the clock is loaded with has passed, the clock generates an interrupt. The TERAK then attempts to execute a nonexistent routine and almost invariablely crashes the system. However, turning off the clock only helps when the program terminates normally. If the execution is aborted, it is almost impossible to catch the clock in its "off" state, and the interrupt is still generated.

This method of timing has served us well. We have collected about 30,000 latencies in a number of different experiments, and once the initial problems were overcome, the hardware and the software have been reliable.

\section{HANDLING LARGE FILES ON A SMALL COMPUTER}

Within our area of research, large amounts of text are required for presentation during a particular experiment. The amount of reading involved for an individual sub. ject, merely for the sake of obtaining one data point, can be up to nine sentences of background text. For example, in one particular study we used a primary text of 900 sentences, with each sentence using an average of 57 characters. This text alone required over $50 \mathrm{~KB}$ of storage.

Two major difficulties are encountered with large files. First, the simple concern of how much disk space is available comes into play, especially when stimuli files typically contain over 100 sentences for each between-subjects condition desired. With the already 
large overhead involved in storing the operating system, the addition of another large chunk for stimuli and background information can severely restrict the amount of data that one is able to store. The other concern is the ability for the files to be reasonably handled by programs and system utilities. In particular, the editor provided by the PASCAL system simply would not edit our text file. Since the text had been typed into our mainframe computer and transferred via a serial line to the microcomputer, this was not a serious problem for the initial creation of the file. But since the re were inevitable typographical errors, as might be expected in a 900 sentence text, editing became a nuisance.

There are several solutions for this problem, ranging from changing the experiment to buying more equip. ment (note the pseudodisk concept used by Fox et al., 1981). Two suggestions are detailed here. The first involves breaking up a single large text into smaller pieces that are manageable by the system; the second involves incorporating a control variable into the procedure.

A particularly large text that must remain contiguous can be troublesome when handled as an individual file, but the file can be broken up into smaller chunks. The accessing experimental program can be designed to take such a situation into account. Each subsection of the text is opened in a specified order, starting with the beginning of the text. When the last file is closed, the actual end of file is reached and can be handled appropriately.

There are several ways of implementing the ordering of the file openings. One such method entails having a fixed list of individual file names that are accessed and opened in turn. The method we chose involved the adjustment of a specific character within one file name. The name of the file for text input was initialized to the name of the first of the group of files that made up the entire text. Further, the names of all files involved differed only in one character, with this character being assigned according to each file in the order of the group (i.e., the totally ordered nature of ASCII was isomorphically mapped to the order of the group of files). The code that used this feature allowed for a simple modification of the construct when only one file is being read. First, the file name that was present at initialization was opened; then, where normally there was an "EOF(file)" statement, a Boolean function was used. In the case of an end of file, if the last file in the group had not already been opened, the function would update the appropriate character in the file name (according to ASCII succession) and then reopen the same file variable. After this had been accomplished, the function would return a true value. This protocol was followed until the last file, as denoted by the last character assigned to the last file of the group. Then the function returned a value of false, indicating that all of the group file had been opened and that the experimental session was over.

This method has worked well for presenting large contiguous texts. However, in research it is not always the case that one must present a lot of information in a specific sequence. Our SBERG program was such a design. Each scenario (up to seven sentences plus one test sentence) made up a small contiguous block that could not be split apart, but, since we had a large number of scenarios ( 280 per subject), the order could be varied. Further, the amount of space required for storing the text material virtually prohibited the creation of data files on the system disk. (Recall that we were using a single-disk system.)

We chose a slightly different method that involved splitting the entire set of scenarios in to five groups. This was done along the number-of-sentences-presented and true-false variables; that is, each group of 56 scenarios had two true and two false situations for each of four sentence set sizes (i.e., one, three, five, and seven). Once these groups were formed, we put each group on a different system disk. Thus, each subject received 56 scenarios on each of five disks, with the experimenter having the added task of changing the system disk periodically. Further, we incorporated the disk presentation order in to the design by using a 5 by 5 Latin square, with subjects as rows, presentation order as columns, and disk numbers as entries. This approach allowed us to incorporate a control variable into the design and reduce the amount of storage per disk, allowing data for several subjects to be stored on each disk. The cost to us was the need for multiple disks, which slightly increased data management responsibilities.

Before leaving the topic of large files, a suggestion is made about the creation of data files. The normal situation encountered in creating data files (i.e., the output of one type of data for each subject) is simple and straightforward. One simply opens a single data file and writes to his satisfaction. The situation is somewhat more complicated when different types of data are to be recorded (e.g., the third version of CONMEM). In a single-disk system, the physical location of a new file is usually at the end of what already exists. When two files are opened, care must be exercised in placing the second file. Some systems allow the program to specify how much room to set aside for each file at opening; this can completely take care of the matter, provided the programmer knows a reasonably safe estimate of how large the files will be. Another solution is to write both types of data to one file and add a code field for specifying the type of data a particular line holds. Then a collating program can be written that summarizes the data according to appropriate type. This can also cut down slightly on storage by reducing the amount of system overhead involved in creating a file. This amount can be significant or minimal, depending on the system.

\section{OTHER CONSIDERATIONS}

\section{Stimulus Control}

The availability within PASCAL of opening sophisticated record types that mix modes of output allows a programmer to write eloquent codes. But using these 
capabilities can be troublesome. The actions of editing a data file, printing it, transferring to a mainframe, and so on, can all involve problems. We found it simpler to write data out using writeln. This function translates variables into text type before outputting, so files have to be opened accordingly. Formats can force data into specific fields, and this is vital if one transfers data to different systems and reads files using formatted languages (e.g., FORTRAN). When reading in a stimulus label that carries information vital to the experimental, a conversion from character to integer or real types can be performed without much difficulty. Typically, the stimuli files are set up with parameter information at the beginning of the record. This information is translated to integer using "ORD" on any single-character substrings that are vital. After abstracting the important information, the "delete" function is used to strip away the parameter section of the record, leaving only the stimulus for presentation.

\section{Data Transfer}

The advantage of transferring data directly from a microcomputer that collects data to a mainframe computer is not unknown to many. Having this capability can eliminate the chore of typing by hand reams of data that have been collected, a tedious and time-consuming task. Briefly, there are four ways of effecting such a connection. The first two require special capabilities of the mainframe computer: using a floppy disk reader on the mainframe and having a parallel port available to the microcomputer. The second two hold that, as far as the mainframe is concerned, the microcomputer acts as little more than a terminal: the use of a serial terminal port (on the mainframe) as either a hardwired or a phone-linked terminal with the microcomputer.

As the first two suggestions require more access to a mainframe computer than the typical microcomputer owner has, they will not be developed further. The second two suggestions are very tractable, however. What is needed at the microcomputer is a serial port and a program that handles the transactions from that side. While it is possible to live with the operating system that a particular mainframe has without writing software to handle it, some kind of program should be developed that can pause when either system's overhead starts to impair the connection. Then either of the two physical connections can be made. If available, the hardwired terminal would be preferable. Here, the microcomputer acts as a regular terminal to the mainframe. The main advantages over a phone link are the higher degree of reliability of transmission due to less noise on the port line and the usually higher baud rate. Phone hookups can be used in the absence of an available hardwire line or when greater flexibility is needed (e.g., transportation away from the laboratory). Modems can be bought and converted to a microcomputer, or, in some cases, terminals can be adapted for such use. The Texas Instruments Silent 700 has this available as an option. It is a relatively simple and straightforward modification.

\section{Screen Manipulation}

The dual-screen setup that the TERAK possesses is very useful for setting up text material and presenting the material on the screen at a high rate. This is accomplished by using the "unitwrite" system intrinsic. This function has a parameter that allows blanking the screen while the text that was or will be displayed remains in the character buffer. When this is not available, it can be effected by an assembler subroutine, providing that the absolute screen buffer address is known. The speed of such a subroutine is sufficient to present an entire screen of text before an eye movement can occur.

\section{Randomization}

The literature of published and available computer programs or randomization can be very helpful by providing mathematical algorithms that simulate random activity. Our program is copied from a demonstration program that was provided with the basic UCSD PASCAL system. Another good source is Ruckdeschel (1981), who provides not only a function for a linear distribution, but also a BASIC language code for adapting such a uniform distribution to nine different random-variable distributions.

When randomization for varying presentation order of stimuli is performed, a uniform distribution should be used so that each stimulus has an equal chance of appearing at any of the possible positions. To do this, one can simply keep generating random numbers and wait for this process to end. A more efficient way to do this, however, is to generate a list of $\mathrm{N}$ random numbers in an array along with a second array that has the integers from 1 to $\mathrm{N}$ in order. Then a simple sort can be applied to the first array, with the equivalent position changes being made in the second array. This results in the second array's containing a random ordering of 1 to $\mathrm{N}$, with the amount of time being regular and small, provided an efficient sort routine, such as a Shell-Metzler, is used.

\section{REFERENCE NOTE}

1. Soloway, E. M., \& Woolf, B. From problems to program via plans: The content and structure of knowledge for introductory LISP programming (COINS Tech. Rep. 80-19). Amherst: University of Massachusetts, Computer and Information Science Department, 1980.

\section{REFERENCES}

Christian, T. L., \& Polson, P. G. The precision of latency measures on real-time computing systems. Behavior Research Methods \& Instrumentation, 1975, 7, 173-178.

Fox, J. L., McKnight, R. J., \& Wolf, R. V. UCSD PASCAL with a VAX as a floppy disk. Behavior Research Methods \& Instrumentation, 1981, 13, 181-188.

Grogono, P. Programming in PASCAL (Rev. ed.). Reading, Mass: Addison-Wesley, 1980.

Post, T. A. Software control of reaction time studies. Behavior Research Methods \& Instrumentation, 1979, 11, 208-211.

Ruckdeschel, F. R. BASIC scientific subroutines (Vol. 1). New York: McGraw-Hill, 1981.

Sternberg, S. The discovery of processing stages. Extensions of Donder's method. Acta Psychologica, 1969, 30, 276-315. 